\title{
Local and Global Region-Based Curve Evolutions for Image Segmentation
}

\author{
Haiyan Zhang ${ }^{1, *}$, Shangbing Gao ${ }^{1,2}$ and Yufu Zhu ${ }^{1}$ \\ ${ }^{I}$ Faculty of Computer Engineering,Jiangsu Provincial KeyLaboratory for Interventional Medical Devices, Huaiyin Insti- \\ tute of Technology, China; ${ }^{2}$ Jiangsu Provincial Key Laboratory for advanced manufacturing technology, Huaiyin Insti- \\ tute of Technology, Huaian, 223003, China
}

\begin{abstract}
In this paper, we propose a novel region-based active contour model in a variational level set formulation. The energy functional for the proposed model consists of two terms, i.e., global term, local term. Therefore, the combination of these two forces allows for flexible initialization of the contours. This energy is then incorporated into a level set formulation with a level set regularization term that is necessary for accurate computation in the corresponding level set method. The proposed model is first presented as a two-phase level set formulation and then extended to a multi-phase formulation. Finally, experiments on some synthetic and real images have demonstrated the efficiency and robustness of our model. Moreover, comparisons with recent popular local image fitting (LIF) model also show that our model is less sensitive to the location of initial contour.
\end{abstract}

Keywords: Active contour, chan-vese, image segmentation, level set.

\section{INTRODUCTION}

Since the introduction by Kass et al. [1], active contour models have been widely used in image segmentation with promising results. The models are able to provide smooth and closed contours to recover object boundaries with subpixel accuracy, which is typically not possible in classical methods, such as edge detection and thresholding. The existing active contour models can be categorized into two classes: edge-based models [1-5] and region-based models [6-10]. The edge-based models utilize image gradient as an additional constraint to stop the contours on the boundaries of desired objects. Usually, a stopping function is used to attract the contours to the desired boundaries. In order to enlarge the capture range of the force, a balloon force term is often incorporated into the evolution function, which controls the contour to shrink or expand. However, it is difficult to choose a proper balloon force. Region-based models aim to identify each region of interest by a certain region descriptor to guide the motion of the active contour. One of the most popular region-based models is the $\mathrm{CV}$ model [6], which has been successfully used in binary phase segmentation with the assumption that each image region is statistically homogeneous. However, the CV model does not work well for the images with intensity inhomogeneity. Vese and Chan extended their work in [9] to utilize multiphase level set functions to represent multiple regions. These models are called the piecewise constant (PC) models. Nonetheless, both the $\mathrm{CV}$ and the PC models have the drawback described above.

Li et al. [7] proposed the LB (local binary fitting) model, which utilizes the local image information as constraints, can well segment objects with intensity inhomogeneities. Furthermore, LBF model has better performance than PC models in segmentation accuracy and computational efficiency. Local image fitting model was recently proposed in [10] which have similar capability of handling intensity inhomogeneity as the LBF model. However, to some extent these methods are still sensitive to initial contour, which holds back their practical applications.

In this paper, we propose an improved region-based active contour model in a variational level set formulation. We use the image fitting model energy functional [10], which is dominant near object boundaries and responsible for attracting the contour toward object boundaries, and an auxiliary global intensity fitting term, which incorporates global image information to improve the robustness of the proposed method. In addition, this energy is then incorporated into a level set formulation with a level set regularization term that is necessary for accurate computation in the corresponding level set method. In the associated curve evolution, the motion of the contour is driven by a local intensity fitting force and a global intensity fitting force, induced by the local and global terms in the proposed energy functional, respectively. The influence of these two forces on the curve evolution is complementary. When the contour is close to object boundaries, the local intensity fitting force becomes dominant, which attracts the contour toward and finally stops the contour at object boundaries. This force plays a key role in accurately locating object boundaries, especially for images with intensity inhomogeneity. The global intensity fitting force is dominant when the contour is far away from object boundaries, and it allows more flexible initialization of contours by using global image information.

The proposed model is first presented as a two-phase level set formulation and then extended to a multi-phase formulation for brain MR image segmentation. The remain- 
der of this paper is organized as follows. In Section 2, we review some well-known region-based models and their limitations. The proposed method is introduced in Section 3. The implementation and results are given in Section 4.

\section{RELATED WORK}

\subsection{Chan-Vese Model}

Chan and Vese [6] proposed an active contour approach to the Mumford-Shah problem for a special case where the original image is a piecewise. For a given image $I$ in domain $\Omega$, the CV model is formulated by minimizing the following energy functional:

$$
\begin{gathered}
E\left(C, c_{1}, c_{2}\right)=\lambda_{1} \int_{\text {inside }(C)}\left|I(x, y)-c_{1}\right|^{2} d x d y+\lambda_{2} \\
\int_{\text {outside }(C)}\left|I(x, y)-c_{2}\right|^{2} d x d y,(x, y) \in \Omega
\end{gathered}
$$

where $c_{1}$ and $c_{2}$ are two constants which are the average intensities inside and outside the contour, respectively. With the level set method, we assume

$$
\begin{aligned}
& \left\{\begin{array}{l}
C=\{(x, y) \in \Omega: \varphi(x, y)=0\}, \\
\text { inside }(C)=\{(x, y) \in \Omega: \varphi(x, y)>0\}, \\
\text { outside }(C)=\{(x, y) \in \Omega: \varphi(x, y)<0\},
\end{array}\right. \\
& c_{1}(\varphi)=\frac{\int_{\Omega} I(x, y) \cdot H(\varphi) d x d y}{\int_{\Omega} H(\varphi) d x d y}, \\
& c_{2}(\varphi)=\frac{\int_{\Omega} I(x, y) \cdot(1-H(\varphi)) d x d y}{\int_{\Omega}(1-H(\varphi)) d x d y}
\end{aligned}
$$

By incorporating the length and area energy terms into Eq. (1) and minimizing them, we obtain the corresponding variational level set formulation as follows:

$$
\frac{\partial \varphi}{\partial t}=\delta(\varphi)\left[\mu \operatorname{div}\left(\frac{\nabla \varphi}{|\nabla \varphi|}\right)-v-\lambda_{1}\left(I-c_{1}\right)^{2}+\lambda_{2}\left(I-c_{2}\right)^{2}\right]
$$

where $\mu \geq 0, v \geq 0, \lambda_{1}>0, \lambda_{2}>0$ are fixed parameters, $\mu$ controls the smoothness of zero level set, and $\lambda_{1}$ and $\lambda_{2}$ control the image data driven force inside and outside the contour, respectively. $\nabla$ is the gradient operator. $H(\phi)$ is the Heaviside function and $\delta(\phi)$ is the Dirac function.
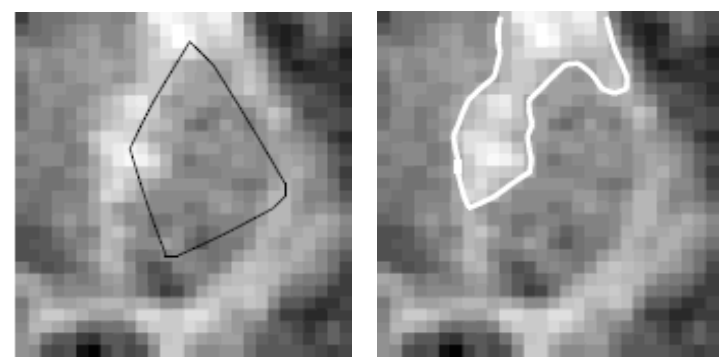

Fig. (1). (a) A 2D slice of a 3D MRI image; (b) the segmentation result of CV model.
Such optimal constants $c_{1}$ and $c_{2}$ can be far different from the original data, if the intensities within outside $(C)$ and inside $(C)$ are inhomogeneous. Without taking local image information into account, the CV model generally fails to segment images with inhomogeneity. It is can be seen in Fig. (1).

\subsection{LIF Model and Its Variational Level Set Formulation}

A local image fitting (LIF) formulation [10] is defined as follows:

$$
I^{L I F}=m_{1} H_{\varepsilon}(\phi)+m_{2}\left(1-H_{\varepsilon}(\phi)\right)
$$

where $m_{1}$ and $m_{2}$ are defined as follows:

$$
\left\{\begin{array}{l}
m_{1}=\operatorname{mean}\left(I \in\left(\{x \in \Omega \mid \phi(x)<0\} \cap W_{k}(x)\right)\right) \\
m_{2}=\operatorname{mean}\left(I \in\left(\{x \in \Omega \mid \phi(x)>0\} \cap W_{k}(x)\right)\right)
\end{array}\right.
$$

where $W_{k}(x)$ is a rectangular window function, e.g. a truncated Gaussian window or a constant window. In our experiment, we choose a truncated Gaussian window $k_{\sigma}(x)$ with standard deviation $\sigma$ and of size $4 k+1$ by $4 k+1$, where $\mathrm{k}$ is the greatest integer smaller than $\sigma$. Similar segmentation results can be achieved if we choose a constant window. In this paper we propose a local image fitting energy functional by minimizing the difference between the fitted image and the original image. The formulation is as follows:

$$
E^{L I F}(\phi)=\frac{1}{2} \int_{\Omega}\left|I(x)-I^{L I F}(x)\right|^{2} d x, x \in \Omega
$$

Using the calculus of variation and the steepest descent method, we minimize $E^{L I F}(\phi)$ with respect to $f$ to get the corresponding gradient descent flow:

$\frac{\partial \phi}{\partial t}=\left(I-I^{L I F}\right)\left(m_{1}-m_{2}\right) \delta_{\varepsilon}(\phi)$

where $\delta_{\varepsilon}(\varphi)=\frac{1}{\pi} \cdot \frac{\varepsilon}{\varepsilon^{2}+z^{2}}, z \in R$.

However, such localization property may introduce many local minimums of the energy functional. Consequently, the result is more dependent on the initialization of contour. This can be seen from a simple experiment for a binary image with a special initial contour as shown in Fig. (2b).
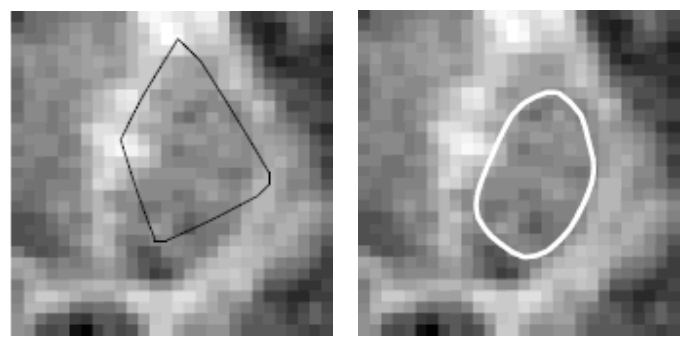
Fig. (2). (a) A 2D slice of a 3D MRI image; (b) the segmentation result of LIF model.

\section{THE NEW METHOD}

Our method combines the advantages of the CV model and the LBF model by taking the local and global intensity information into account. In this section, we will detail our active contour model based on local and global region-based energy for image segmentation. The overall energy functional consists of three parts: global term $E_{G}$, local term $E_{L}$ and regularization term $E_{R}$.

\subsection{The Global Term}

Accordingly, the global term in (1) can be rewritten so as to evaluate the level set function $\phi$ on the domain $\Omega$ :

$$
\begin{aligned}
& E^{G}=\int_{\Omega}\left|I(x, y)-c_{1}\right|^{2} H(\varphi(x, y)) d x d y \\
& +\int_{\Omega}\left|I(x, y)-c_{2}\right|^{2}(1-H(\varphi(x, y)) d x d y
\end{aligned}
$$

\subsection{The Local Term}

Here, the local term is introduced in (7) which uses the local statistical information to improve the segmentation capability of our model for the images with intensity inhomogeneity.

$$
\begin{aligned}
& E^{L}=\frac{1}{2} \int_{\text {inside }(C)}\left|I(x)-I^{L I F}(x)\right|^{2} d x d y \\
& +\frac{1}{2} \int_{\text {outside }(C)}\left|I(x)-I^{L I F}(x)\right|^{2} d x d y
\end{aligned}
$$

\subsection{The Regularization Term}

It is crucial to keep the evolving level set function as an approximate signed distance function during the evolution, so in the MCV model, re-initialization is used as a numerical remedy for maintaining stable curve evolution as follows:

$\frac{\partial \phi}{\partial t}=\operatorname{sign}\left(\phi_{0}\right)(1-|\nabla \phi|)$

But it leads very complicated process and side effects, so we use the following integral:

$$
p(\phi)=\int_{\Omega} \frac{1}{2}\left(|\nabla \phi(x, y)-1|^{2}\right)
$$

The above expression is formulated as a metric to characterize how close the function $\phi$ is to the signed distance function. By minimizing the above expression, $|\phi|$ can be made to converge to 1 , so that the level set function is made close to the signed distance function.

According to the above words, the regularized term is expressed as follows:

$$
\begin{aligned}
& E^{R}=\int_{\Omega} \frac{1}{2}\left(|\nabla \varphi(x, y)-1|^{2}\right) d x d y+\mu \cdot \text { Length }(C)= \\
& \int_{\Omega}\left(\frac{1}{2}\left(|\nabla \phi(x, y)-1|^{2}\right)+\mu \cdot \delta_{\varepsilon}(\phi)|\nabla \phi|\right) d x d y
\end{aligned}
$$

where $v$ increases the propagation speed.

\subsection{Level Set Formulation}

In the level set formulation, the curve $\mathrm{C}$ is represented by the zero level set of a Lipschitz function $\phi$. The overall energy functional can be described as follows:

$$
\begin{aligned}
& E^{G}=E^{G}+E^{L}+E^{R}=\omega\left(\int_{\text {inside }(C)}\left|I(x, y)-c_{1}\right|^{2} H(\varphi(x, y)) d x d y\right. \\
& +\int_{\text {outside }(C)}\left|I(x, y)-c_{2}\right|^{2}(1-H(\phi(x, y)) d x d y)+(1-w) \\
& \left(\frac{1}{2} \int_{\text {inside }(C)}\left|I(x)-I^{L I F}(x)\right|^{2} d x d y+\frac{1}{2} \int_{\text {outside }(C)}\left|I(x)-I^{L I F}(x)\right|^{2} d x d y\right) \\
& +\int_{\Omega}\left(\frac{1}{2}\left(|\nabla \varphi(x, y)-1|^{2}\right)+\mu \cdot \delta_{\varepsilon}(\varphi)|\nabla \varphi|\right) d x d y
\end{aligned}
$$

where $w$ is a positive constant $(0 \leq w \leq 1)$. When the images are corrupted by intensity inhomogeneity, the parameter value $w$ should be chosen small enough.

As shown in Fig. (3), if $\varepsilon$ is too small, the values of $\delta_{\varepsilon}(z)$ tend to be near zero to make its effective range small, so the energy functional has a tendency to fall into a local minimum. The object may fail to be extracted if the initial contour starts far from it. However, if $\varepsilon$ is large, although $\delta_{\varepsilon}(z)$ tends to obtain a global minimum, the finial contour location may not be accurate. So in this paper, we choose $\varepsilon=1.5$.

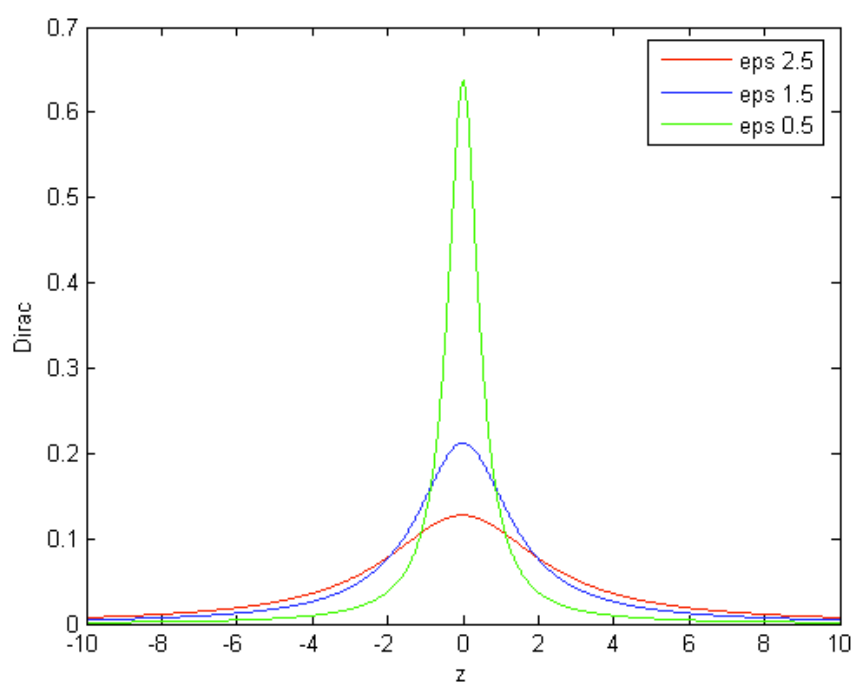

Fig. (3). The Dirac function w.r.t different epsilon values. 

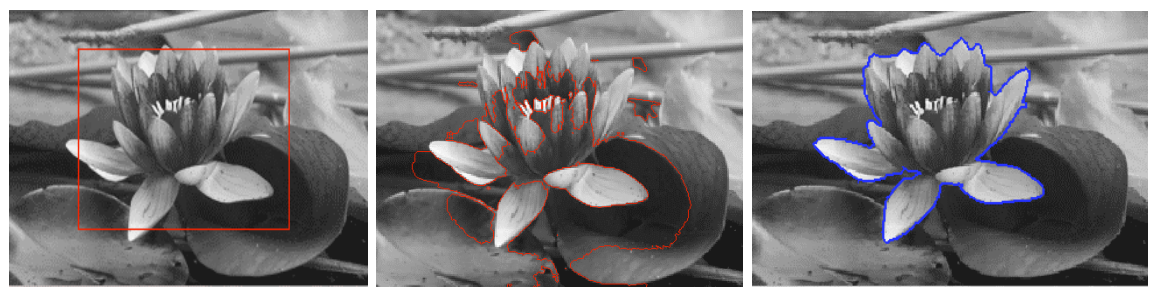

Fig. (5). (a) flower image (b) the segmentation result of LIF model; (c) the segmentation result of the proposed model.
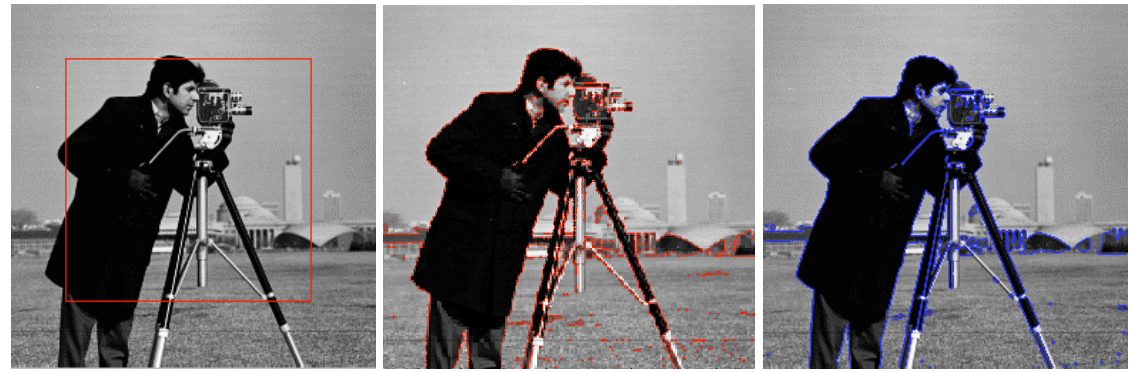

Fig. (6). (a) The cameraman image (b) The segmentation result of LIF model (c) The segmentation result of the proposed model.
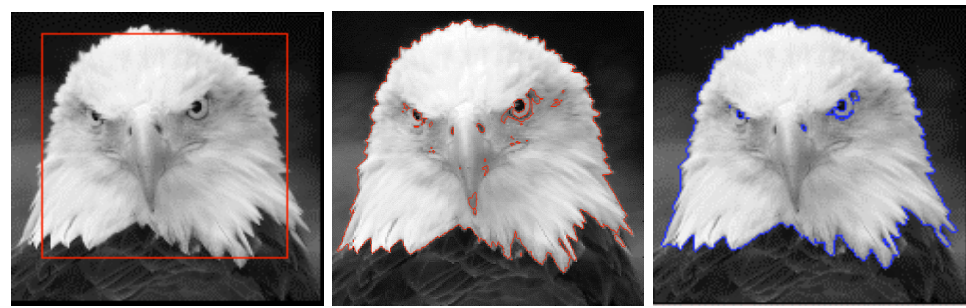

Fig. (7). (a) The eagle image (b) The segmentation result of LIF model (c) The segmentation result of the proposed model.

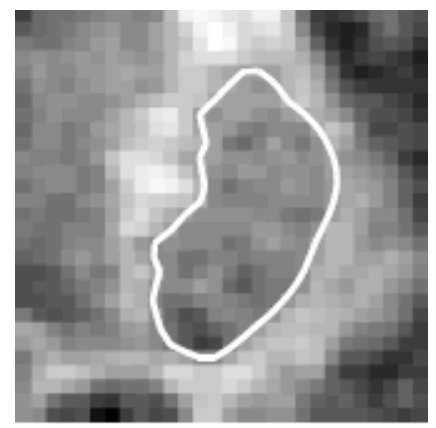

Fig. (4). The correct result of the proposed method.

The corresponding result of the proposed method of Fig. (1a) is shown in Fig. (4).

\section{EXPERIMENTS}

In this section, we apply the proposed model to images to demonstrate its effectiveness. In each experiment, we select the parameters as follows: $\mu=1, \quad v=0.001 * 255 * 255$, $c_{0}=2, \sigma=3$.

To demonstrate the advantages of our model in terms of accuracy, we compare it with the LIF model [9]. Form the below figures, we can see that our model outperforms the LIF model.

Fig. (5a) is an original flower image, is the original image (a), (b) is the segmentation result of LIF model, its itera- tion is 200 times, (c) is the segmentation result of our method. Fig. (5b) shows that the LIF model takes some object areas which gray value is similar to background areas as the background area. And LIF model is unable to set the number of iterations according to the size of the image, leading to fail to completely segment after finite iterations. As can be seen from the Fig. (5c), our method can obtain the object boundary correctly, and does not need to adjust the number of iterations for different images.

Fig. (6a) is an original portrait photograph of people image, (b) is the segmentation result of LIF model, its iteration is 200 times, (c) is the segmentation result of our method. LIF model segment the background region especially the grass part as the object. However, our model can extract the portrait and the main object of the similar gray value.

Fig. (7a) is an original hawk image, (b) is the segmentation result of LIF model, its iteration is 200 times, (c) is the segmentation result of our method. LIF model segments the eagle head beak as the object, but the phenomenon of excessive segmentation happens. However, our model overcomes the effect of excessive segmentation so as to extract the complete object boundary.

Fig. (8) shows an experiment on the chosen brain image with our method. Inhomogeneity of the image intensity can be clearly seen in Fig. (8a). The proposed method and the LIF method are extended to the multiphase model according to [11]. It can be clearly seen that the multiphase LIF model 


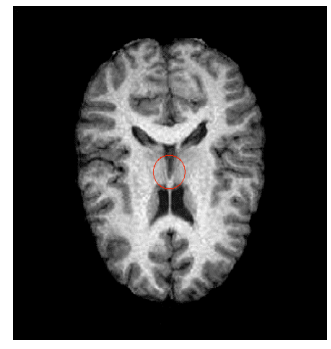

(a) The initial contour

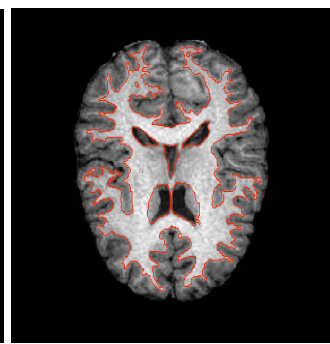

(b) The segmentation result of multiphase LIF model

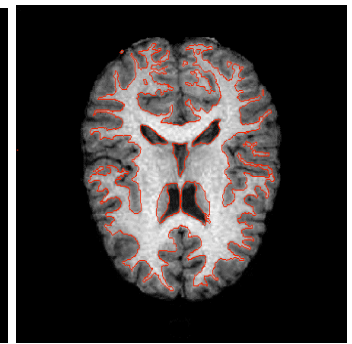

(c) The segmentation result of the proposed model

Fig. (8). The segmentation comparison between the proposed model and LIF method.

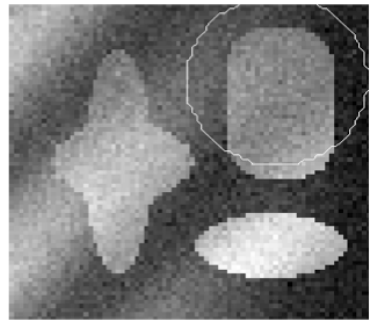

(a) Initi al contour

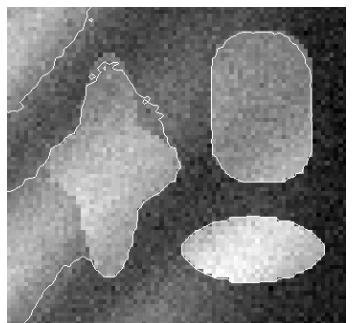

(b) CV model

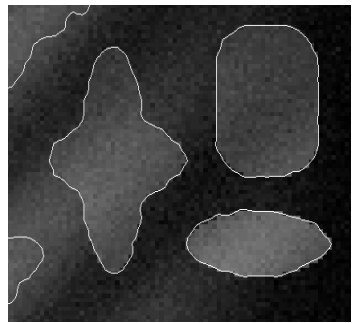

(c) LIF model

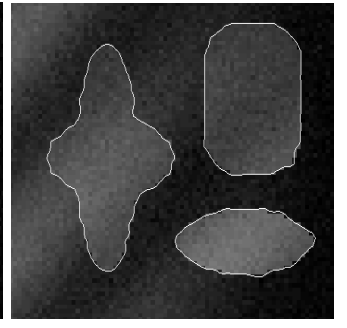

(d) Our model

Fig. (9). The comparison of the segmentation results of three models.

does not correctly segment images. By contrast, our method recovers the boundaries of WM, GM, and CSF accurately.

In order to further verify the Segmentation result of our model, we use the more complex and noisy image, as shown in Fig. (2). As can be seen in Fig. (2), the gray value discretion is striped, respectively, and the gray value of the object in the dark streak region is smaller than the gray value of background light stripe region.

In this paper, we use the initial contour in Fig. (9a) to carry out the segmentation experiments with the three models respectively. The segmentation results are shown in Fig. (9b-d). From Fig. (9b-d), it can be seen that the CV model and LIF model can't correctly segment image, but our model can segment image accurately. Although the LIF model considers the local features, the model is sensitive to initial contour, thus the LIF model can't correctly segment image. Table 2 shows the iteration times and running time of three models. As can be seen from Table 2, the number of iterations model and running time of $\mathrm{CV}$ is minimum, the number of iterations model and running time of LIF model is the maximum. Furthermore, CV model and LIF model can correctly segment images. The number of iterations and running time is smaller than LIF model. It is because that LIF model only uses the local characteristic of the image, our model in the early period takes advantage of the fast convergence and non-sensitive to the initial contour of the CV model. Therefore, our model can correctly image segmentation, and needs less running time.

\section{CONCLUSION}

This paper takes advantage of the fast convergence and the non-sensitivity on the initial contour of CV model, the accurately inhomogeneous image segmentation LIF model to put forward the regional adaptive active contour model. This model defines a global energy term and a local energy functional term. At the beginning of the contour evolution, the global energy dominants, it has the fast convergence rate and is not sensitive to initial contour position. In the late evolution, local energy dominants, it has the advantages of high positioning accuracy. Compared with CV model and the LIF model, our model can not only correctly segment the inhomogeneous images, but also has fast convergence rate and is not sensitive to initial contour.

\section{CONFLICT OF INTEREST}

The authors confirm that this article content has no conflict of interest.

\section{ACKNOWLEDGEMENTS}

This work is sponsored by the National Natural Science Foundation of China (NSFC) \#61402192, JiangSu Qing Lan Project, the open fund of Jiangsu Provincial key laboratory for interventional medical devices (JR1405), six talent peaks project in Jiangsu Province, Jiangsu 333 Project and supported in part by the Major Program for scientific and technological research in University of China under the Grant No.311024, Huai'an science and technology support plan project (HANZ2014006), and the open fund of Jiangsu Provincial Key Laboratory for advanced manufacturing technology (HGAMTL-1401).

\section{REFERENCES}

[1] M. Kass, A. Witkin, D." Terzopoulos, Snakes: active contour models", Int'1 J. Comp. Vis., 1987,1 (4): 321-331.

[2] Shangbing Gao, Jian Yang, Yunyang Yan." A Local Modified ChanVese Model for Segmenting Inhomogeneous Multiphase Images”. International Journal of Imaging Systems and Technology,2012, 22:103-113. 
[3] Yunyang Yan, Shangbing Gao, Suqun Cao. "Active contour based Natural Color Images segmentation". Journal of Computational Information Systems, 2012,8(9):3707-3714.

[4] L. Rada, K. Chen,"A new variational model with dual level set functions for selective segmentation", Communications in Computational Physics, 2012,12 (1):261-283.

[5] L. Cohen and I. Cohen, "Finite-element methods for active contour models and balloons for 2-D and 3-D images", IEEE Transactions on Pattern Analysis and Machine Intelligence, 1993,15(11):11311147.

[6] D. Sen, S.K. Pal, "Improving feature space based image segmentation via density modification", Information Sciences,2012,19(115): 69-191.
[7] C. Li, C. Kao, J. Gore, Z. Ding, "Minimization of region-scalable fitting energy for image segmentation", IEEE Transactions on Image Processing, 2008, 17:1940-1949.

[8] Shangbing Gao, Yunyang Yan. "Brain MR Image Segmentation via a Multiphase Level Set Approach, Journal of Information Computational Systems", 2012:214-218

[9] I. Posirca, Y. Chen, C.Z. Barcelos, "A new stochastic variational PDE model for soft Mumford-Shah segmentation", Journal of Mathematical Analysis and Applications, 2011,384 (1) :104-114

[10] K. Zhang, H. Song, L. Zhang," Active contours driven by local image fitting energy". Pattern Recognition, 2010, 43(4): 11991206.

(C) Zhang et al.; Licensee Bentham Open.

This is an open access article licensed under the terms of the Creative Commons Attribution Non-Commercial License (http://creativecommons.org/licenses/by-nc/3.0/) which permits unrestricted, non-commercial use, distribution and reproduction in any medium, provided the work is properly cited. 